\title{
Plasma linoleic acid levels and cardiovascular risk factors: Results from the Norwegian ACE 1950 Study
}

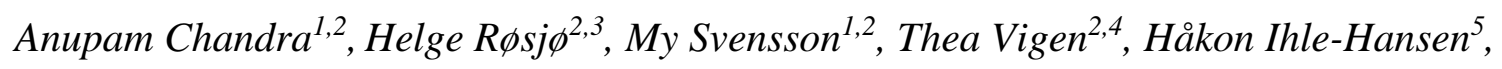
Eivind Bjфrkan Orstad ${ }^{4}$, Ole Morten Rønning ${ }^{2,4}$, Magnus Nakrem Lyngbakken $^{2,4}$, Ståle Nygård ${ }^{6,7}$, Trygve Berge ${ }^{5}$, Erik Berg Schmidt ${ }^{8}$, Torbjørn Omland ${ }^{2,4}$, Arnljot Tveit $^{2,5}$, Ivar Anders Eide ${ }^{l}$.

${ }^{1}$ Department of Renal Medicine, Division of Medicine, Akershus University Hospital, Lørenskog, Norway.

${ }^{2}$ Institute of Clinical Medicine, University of Oslo, Norway.

${ }^{3}$ Division of Research and Innovation, Akershus University Hospital, Lørenskog, Norway.

${ }^{4}$ Division of Medicine, Akershus University Hospital, Lørenskog, Norway.

${ }^{5}$ Department of Medical Research, Bærum Hospital, Vestre Viken Hospital Trust, Drammen, Norway.

${ }^{6}$ Centre for Bioinformatics, Department of Informatics, University of Oslo, Oslo, Norway.

${ }^{7}$ Oslo Centre for Biostatistics and Epidemiology, Oslo University Hospital, Oslo, Norway.

${ }^{8}$ Department of Cardiology, Aalborg University Hospital, Aalborg, Denmark.

\section{Corresponding author:}

Anupam Chandra, Department of Renal Medicine, Division of Medicine, Akershus

University Hospital, Sykehusveien 25, 1478 Lørenskog, Norway. Anupam.Chandra@ahus.no, +4793818386. 


\section{Running title:}

Linoleic acid and cardiovascular risk factors

\section{Abbreviations:}

ACE

BMI

cIMT

CI

CRP

CV

$\mathrm{DM}$

eGFR

HbA1c

HDL

LA

LDL

PUFA

Std. $\beta$-coeff.

Unstd. $\beta$-coeff.

$\mathrm{wt} \%$
Akershus Cardiac Examination

Body mass index

Carotid intima-media thickness

Confidence interval

C-reactive protein

Cardiovascular

Diabetes mellitus

Estimated glomerular filtration rate

Glycated hemoglobin

High-density lipoprotein

Linoleic acid

Low-density lipoprotein

Polyunsaturated fatty acid

Standardized regression coefficient

Unstandardized regression coefficient

Weight percentage 


\begin{abstract}
Background: A high intake of linoleic acid (LA), the major dietary polyunsaturated fatty acid (PUFA), has previously been associated with reduced cardiovascular (CV) morbidity and mortality in observational studies. However, recent secondary analyses from clinical trials of LA-rich diet suggest harmful effects of LA on CV health.
\end{abstract}

Methods: A total of 3,706 participants, all born in 1950, were included in this cross-sectional study. We investigated associations between plasma phospholipid levels of LA and CV risk factors in a Norwegian general population, characterized by a relative low LA and high marine n-3 PUFA intake. The main statistical approach was multivariable linear regression. Results: Plasma phospholipid LA levels ranged from 11.4 to $32.0 \mathrm{wt} \%$, with a median level of $20.8 \mathrm{wt} \%$ (interquartile range 16.8-24.8 wt\%). High plasma LA levels were associated with lower serum low-density lipoprotein cholesterol levels (Standardized regression coefficient [Std. $\beta$-coeff.] -0.04, $\mathrm{p}=0.02$ ), serum triglycerides (Std. $\beta$-coeff. $-0.10, \mathrm{p}<0.001)$, fasting plasma glucose (Std. $\beta$-coeff. $-0.10, \mathrm{p}<0.001$ ), body mass index (Std. $\beta$-coeff. $-0.13, \mathrm{p}<0.001$ ), systolic and diastolic blood pressure (Std. $\beta$-coeff. $-0.04, p=0.03$ and Std. $\beta$-coeff. -0.02 , $\mathrm{p}=0.02$, respectively) and estimated glomerular filtration rate (Std. $\beta$-coeff. $-0.09, \mathrm{p}<0.001)$. We found no association between plasma LA levels and high-density lipoprotein cholesterol levels, glycated hemoglobin, carotid intima-media thickness or C-reactive protein.

Conclusion: High plasma LA levels were favorably associated with several CV risk factors in this study of a Norwegian general population. 


\section{Introduction}

Linoleic acid (LA) is an essential fatty acid, mainly derived from vegetable oils, nuts, eggs and meats (1). It is the major n-6 polyunsaturated fatty acid (PUFA) and the most abundant PUFA in the Western diet (1). LA intake has previously been associated with a lower risk of cardiovascular (CV) morbidity and mortality in large epidemiological studies (2-4). However, recent published secondary analysis from the Sydney Diet Heart Study and Minnesota Coronary Experiment have raised concerns, as they report a direct association between diets rich in LA and an increase in overall and coronary heart disease mortality $(5,6)$. One proposed mechanism for this observation is an LA-induced increase in oxidized low-density lipoprotein (LDL) cholesterol particles, which are highly atherogenic $(5,6)$. LA has also been suggested to promote inflammation by serving as a precursor for arachidonic acid, an n-6 PUFA that is oxidized to proinflammatory cytokines during eicosanoid metabolism (7). In addition, LA reduces the conversion of plant derived n-3 PUFA alpha-linolenic acid to the anti-inflammatory n-3 PUFAs eicosapentaenoic and docosahexaenoic acid (7), also found in fatty fish and other seafoods.

The effects of LA intake on CV health have therefore been a matter of discussion and have resulted in inconsistent recommendations on LA consumption across regions. While the American Heart Association recommends consumption of at least 5\% to 10\% energy from LA intake, the European Food Safety Authority, supported by the French national guidelines, recommend 4\% (8-10). A recent systemic review, evaluating LA intake in various European nations, showed that the European Food Safety Authority recommendations were not met in half of the countries included in the study (11). Compared to a modern Western diet, the traditional Nordic diet is characterized by a relatively high marine n-3 PUFA and low LA consumption (12). 
Thus, there is a possibility that the LA intake might be lower than optimal also in Norway. The average energy intake from total PUFAs (the sum of n-3 PUFAs and n-6 PUFAs) was approximately $6 \%$ in Norway in 2012 (13), hence the energy intake from LA alone was probably lower than American Heart Association's recommendations. The assessment of how a marker of LA consumption is associated with the CV risk profile in a Norwegian general population might add new knowledge on the relationship between LA intake and CV health. The objective of this study was to investigate associations between plasma LA levels, as an estimate of LA intake $(14,15)$, and multiple CV risk factors.

\section{Materials and methods}

\section{Study population}

The Akershus Cardiac Examination (ACE) 1950 Study is a prospective population-based cohort study, evaluating the cardio- and cerebrovascular health of men and women born in 1950 and living in Akershus County, Norway. The study design and baseline examinations of the ACE 1950 Study have previously been described in detail (16). In brief, the Norwegian population register was used to identify all eligible individuals in Akershus County, who were invited for study participation by letters and subsequent phone calls. Among 5,827 invited individuals, 3,706 agreed to participate (64\%) (Figure 1). The remaining 2,121 did not respond to the study invitation letter or declined participation. Enrollment was performed at Akershus University Hospital and Bærum Hospital from September 2012 through May 2015. In this article, we present data from a cross-sectional analysis of the baseline examination.

\section{$\underline{\text { Data collection and procedures }}$}

All participants completed questionnaires on medical history, diet, education, physical activity and smoking. Higher education was defined as $>12$ years of formal education. 
Smoking habits were classified as current smoker or previous/non-smoker. High physical activity was defined as $>2$ sessions of exercise per week. Alcohol consumption was defined as intake of alcohol $\geq 2$ times per week. Study participants underwent a standardized physical examination. Hypertension was defined as mean systolic blood pressure $\geq 140 \mathrm{mmHg}$ or mean diastolic blood pressure $\geq 90 \mathrm{mmHg}$ from three measurements in the sitting position, or current use of anti-hypertensive medication. The World Health Organizations definition was used to define obesity (body mass index [BMI, $\left.\mathrm{kg} / \mathrm{m}^{2}\right] \geq 30$ ) (17).

Blood samples were obtained after overnight fasting and stored at $-80{ }^{\circ} \mathrm{C}$ until analysis. Hypercholesterolemia was defined as total serum cholesterol $\geq 6.2 \mathrm{mmol} / \mathrm{L}$ or LDL cholesterol $\geq 4.1 \mathrm{mmol} / \mathrm{L}$ (18), or current use of lipid-lowering agents. Diabetes mellitus (DM) was defined as both glycated hemoglobin $(\mathrm{HbA1c}) \geq 6.5 \%$ and fasting plasma glucose $\geq 7.0 \mathrm{mmol} / \mathrm{L}$, self-reported DM or current use of glucose-lowering medications. Chronic kidney disease stages 3-5 were defined as estimated glomerular filtration rate (eGFR) $<60$ $\mathrm{ml} / \mathrm{min} / 1.73 \mathrm{~m}^{2}$ using The Chronic Kidney Disease Epidemiology Collaboration equation (19). Standard assay was used for the measurement of C-reactive protein (CRP).

Carotid intima-media thickness (cIMT) was assessed by ultrasound examination of the right and left carotid arteries, as previously described (20). Mean cIMT was obtained from the average of right and left cIMT measurements.

\section{Plasma phospholipid fatty acid measurement}

Aliquots from stored frozen blood samples were sent to The Lipid Research Center, Aalborg University Hospital for plasma fatty acid analysis by gas chromatography. In brief, Folch method was used to extract total lipids from serum (21), and the Burge method was used to isolate the phospholipid fraction from other lipids (22). The fatty acids were analyzed by a Varian 3900 gas chromatograph (Varian, Middleburg, The Netherlands) with $60 \mathrm{~m}$ x $0.25 \mathrm{~mm}$ 
capillary columns. Individual fatty acids were identified from their retention time during gas chromatography, and quantified as weight percentage (wt $\%$ ) of total plasma phospholipid fatty acids.

Data on plasma LA levels were missing in 23 participants, either due to insufficient plasma volume $(n=17)$ or inadequate plasma fatty acid analysis $(n=6)$ (Figure 1$)$.

\section{$\underline{\text { Ethical considerations }}$}

All participants gave written informed consent before final enrollment. The study was approved by the Norwegian Regional Ethics Committee (September $7^{\text {th }} 2011$. Ref. number 2011/1475) and performed in accordance with the Declaration of Helsinki. Registered at clinicaltrials.gov, registration number NCT01555411.

\section{$\underline{\text { Statistical analysis }}$}

Quartiles of plasma LA were used for presentation of demographic and clinical data. Results are presented as mean values (standard deviations) for normally distributed data, median values (inter quartile ranges) for skewed data (serum triglycerides, fasting plasma glucose, HbA1c and CRP) and percentages for categorical data. Between-groups differences were assessed by Chi square test for dichotomous variables, Kruskal-Wallis test for skewed variables and ANOVA for normally distributed continuous variables. Bivariate associations between plasma LA levels and plasma eicosapentaenoic and docosahexaenoic acid levels, higher educational level, alcohol consumption, gender, smoking and physical activity are presented with Spearman's correlation $\left(\mathrm{r}_{\mathrm{s}}\right)$. We investigated multivariable adjusted associations between plasma LA levels and CV risk factors, after testing for linearity with standard methods and restricted cubic spline modelling, the graphical presentation for which is presented in Figure 2. For restricted cubic spline modelling, the continuous variable plasma 
LA levels was subdivided by use of three knots, placed at $10^{\text {th }}, 50^{\text {th }}$ and $90^{\text {th }}$ percentile of the exposure distribution. Although assumptions for linearity between plasma LA levels and serum LDL cholesterol and $\mathrm{HbA1c}$ were not fully met, multivariable linear regression was performed for these variables. Adjustments were made with for each CV risk factor with covariates chosen from a set of predefined candidate variables by least absolute shrinkage and selection operator (LASSO) regression (Supplementary table 1). First highly inflated Std. $\beta$ coeff. were penalized towards zero. A tuning parameter lambda, which was chosen for each $\mathrm{CV}$ risk factor after cross validation, controlled the strength of penalty. Second, during variable selection, candidate variables with regression coefficients $>0$ after the shrinkage process, were included in the final model. All models were constructed using simultaneous entry of variables. Skewed variables were truncated to obtain normal distribution, before they were entered into the regression models. Because of extreme skewness, CRP was logarithmically transformed before it was entered as a variable in the regression analyses. Thus, for CRP, the presented unstandardized regression coefficients (Unstd. $\beta$-coeff.) and corresponding 95\% confidence intervals (CI) represent the anti-logarithm of obtained results. The table displays Unstd. $\beta$-coeff. with corresponding 95\% CI, standardized regression coefficients (Std. $\beta$-coeff.), $\mathrm{p}$-values and explained variance $\left(\mathrm{R}^{2}\right)$ for the univariable and the fully adjusted final models. All statistical calculations were performed using SPSS® version 25.0 (IBM, NY, US), RStudio® version 1.1.419 and R version 3.4.4 (RStudio Inc., Boston, MA, US and R Foundation for Statistical Computing, Vienna, Austria).

\section{Results}

\section{$\underline{\text { Demographics }}$}

Demographics and clinical characteristics of study participants are presented in Table 1.

Plasma LA levels ranged from 11.4 to $32.0 \mathrm{wt} \%$, with a median level of $20.8 \mathrm{wt} \%$ 
(interquartile range 16.8 to $24.8 \mathrm{wt} \%$ ). Participants with high plasma LA levels had lower plasma levels of eicosapentaenoic and docosahexaenoic acid $\left(r_{s}=-0.44\right.$ and $r_{s}=-0.37$, $\mathrm{p}<0.001$ for both). Higher education $\left(\mathrm{r}_{\mathrm{s}}=0.07, \mathrm{p}<0.001\right)$ and less alcohol consumption $\left(\mathrm{r}_{\mathrm{s}}=-\right.$ 0.07, $\mathrm{p}<0.001)$ were seen in participants with high plasma LA levels, while no differences were observed for gender $\left(\mathrm{r}_{\mathrm{s}}=-0.01, \mathrm{p}=0.62\right)$, smoking $\left(\mathrm{r}_{\mathrm{s}}=-0.03, \mathrm{p}=0.09\right)$ or physical activity $\left(\mathrm{r}_{\mathrm{s}}=0.03, \mathrm{p}=0.13\right)$. Furthermore, a lower prevalence of hypertension, hypercholesterolemia, cerebrovascular and coronary artery disease, DM and obesity were also seen in participants with high compared to low levels of plasma LA. Moreover, the use of antihypertensive-, lipid-lowering and glucose-lowering medications were lower in participants with higher plasma LA levels.

\section{Plasma LA levels and CV risk factors}

Cubic spline modeling of associations between plasma LA levels and CV risk factors are presented in Figure 2. Except for serum LDL-cholesterol levels and HbA1c, the associations between plasma LA levels and CV risk factors were linear. Unadjusted and multivariable adjusted associations between plasma LA levels and CV risk factors are presented in Table 2. High plasma LA levels were associated with lower serum LDL cholesterol and triglyceride levels, while no association was found with serum high-density lipoprotein (HDL) cholesterol levels.

High plasma LA levels were associated with lower fasting plasma glucose. When stratifying participants according to DM, plasma LA levels showed a statistically significant association fasting plasma glucose in participants without DM ( $\mathrm{n}=3296$, Unstd. $\beta$-coeff. -0.03 [95\% CI $0.03,-0.02]$, Std. $\beta$-coeff. $-0.13, \mathrm{p}<0.001)$, while a trend was seen in participants with DM $(\mathrm{n}=309$, Unstd. $\beta$-coeff. -0.05 [95\% CI $-0.10,0.02]$, Std. $\beta$-coeff. $-0.11, \mathrm{p}=0.15)$. We found no associations between plasma LA levels and HbA1c. 
Plasma LA levels were inversely associated with BMI and systolic and diastolic blood pressure (Table 2).

High plasma LA levels were associated with lower eGFR. Participants were stratified according to eGFR levels above or below $60 \mathrm{ml} / \mathrm{min} / 1.73 \mathrm{~m}^{2}$. Plasma LA levels were inversely associated with eGFR $>60 \mathrm{ml} / \mathrm{min} / 1.73 \mathrm{~m}^{2}$ ( $\mathrm{n}=3452$, Unstd. $\beta$-coeff. -0.28 [95\% CI $0.40,-0.15]$, Std. $\beta$-coeff. $-0.08, \mathrm{p}<0.001)$. In contrast, no association was found with eGFR $<60 \mathrm{ml} / \mathrm{min} / 1.73 \mathrm{~m}^{2}$ (n=141, Unstd. $\beta$-coeff. -0.01 [95\% CI -0.47, 0.46], Std. $\beta$-coeff. -0.003 , $\mathrm{p}=0.98)$

We found no statistical association between plasma LA levels and cIMT or CRP (Table 2).

When stratifying participants according to educational level and alcohol consumption, associations between plasma LA levels and CV risk factors did not change (data not shown).

\section{Discussion}

The main finding of the present study was that high levels of plasma LA were associated with lower fasting plasma glucose and BMI. High plasma LA levels were associated with lower serum triglyceride levels and systolic blood pressure. We found no association between plasma LA levels and CRP.

\section{Plasma LA levels and CV risk factors}

\section{Plasma LA levels and fasting plasma glucose, HbA1c and BMI}

In the present study, we found a lower prevalence of DM among participants in the upper quartile of plasma LA levels. High levels of plasma LA were associated with lower fasting plasma glucose in individuals without DM, while no statistically significant association was 
found in participants with DM, in whom most received glucose-lowering medication that could possibly confound results. In a pooled analysis of 20 prospective studies, including $>39,000$ participants from 10 different countries, high plasma levels of LA were associated with a lower risk of incident type 2 DM (23). These reports, along with our findings, may suggest that LA consumption might contribute in prevention of DM. Surprisingly, plasma LA levels were not associated with $\mathrm{HbA1c}$. Similar results have been reported in a cross-sectional study from the Dutch general population, aged 50-74 (24). Concordant with our findings, they found that high plasma LA levels were associated with lower fasting plasma glucose, while no associations were found with HbA1c (24). The underlying mechanism by which LA influences the glucose-insulin homeostasis is poorly understood. Improved insulin sensitivity mediated by LA has been suggested (25). Another possible mechanism could be increased glucose consumption related to the upregulation of carnitine shuttle system required for mitochondrial $\beta$-oxidation of long-chain fatty acids, such as LA (26). Increased mitochondrial $\beta$-oxidation after a high intake of long-chain fatty acids has also been suggested to induce weight loss (27), which could partly explain the lower prevalence of obesity and lower BMI associated with high plasma LA levels in the present study. On the other hand, weight loss achieved by increased LA intake in clinical trials has been at the expense of energy from carbohydrates (27), and can therefore not be attributed to LA intake alone.

In addition to LA consumption, plasma LA levels are influenced by delta- 6 desaturase activity regulating the endogenous LA metabolism (28). In a population-based cohort study, participants with metabolic syndrome and insulin resistance had higher delta- 6 desaturase activity and lower serum LA levels compared to control (29). A higher delta-6 desaturase activity has also been associated with insulin resistance (30). Hence, we cannot rule out 
reverse causality bias that could possibly underlie inverse associations between plasma LA levels and fasting plasma glucose levels.

\section{Plasma LA levels and CRP}

A concern for harm from a high LA intake has been linked to the potential conversion of LA to arachidonic acid, a precursor for inflammatory cytokines. However, this endogenous conversion is tightly regulated and has been reported to be less than $0.3 \%$ in a tracer study with stabile isotopes (31). In a systematic review of human trials, a 6-fold increase in LA consumption did not affect plasma or tissue concentration of arachidonic acid (32).

Furthermore, a high LA intake in clinical trials did not increase inflammatory biomarkers such as CRP, interleukin-6, tumor necrosis factor- $\alpha$ or plasma fibrinogen (33). In the present study, plasma LA levels were not associated with CRP. Thus, our finding does not support the proposed proinflammatory nature of LA. It should however be mentioned that we did not measure high-sensitive CRP, which is a more reliable marker of low-grade inflammation, hence our finding should be interpreted with caution with regards to CRP levels. Furthermore, the median CRP level was $1.5 \mathrm{mg} / \mathrm{L}$ in our cohort, a level which has been associated with an average risk of CV disease among Caucasians in other community-based studies (34).

Advice to reduce LA intake has also been based on the competition with n-3 PUFAs, as LA theoretically can inhibit the conversion of alpha-linolenic acid to eicosapentaenoic acid and docosahexaenoic acid (7). However, in humans this conversion is low $(<5 \%)(35)$, and likely of minor importance in a Norwegian general population with a high background consumption of marine n-3 PUFAs and a low alpha-linolenic acid intake (12).

\section{Plasma LA levels and serum lipids}


The relationship between LA and blood lipids is not clearly understood. In a meta-analysis of 60 clinical studies, the substitution of LA for carbohydrates reduced serum LDL cholesterol and triglyceride levels (36). Similarly, substituting saturated fatty acids with LA, in a clinical trial in men with dyslipidemia, resulted in a decreased production of LDL particles (37). These reports indicate possible lipid-lowering properties of LA, but should be interpreted with caution as they were obtained at the expense of carbohydrates and saturated fatty acids. One suggested mechanism by which LA influences blood lipids is increased clearance of triglyceride rich particles, the upstream of LDL cholesterol production (37). Furthermore, a high intake of LA has shown to increase lipoprotein lipase activity in experimental studies (38), an enzyme involved in hydrolysis of triglycerides. In the present study, high plasma LA levels were associated with lower serum LDL cholesterol and triglyceride levels.

Furthermore, participants in the upper compared with the lower quartile of plasma LA levels had a lower prevalence of hypercholesterolemia and used lipid-lowering drugs less often. However, the inverse associations between plasma LA levels and serum LDL cholesterol in the present study must be interpreted with caution for several reasons. First, assumptions for a linear association between plasma LA and serum LDL cholesterol levels were not met.

Second, participants in the upper compared to lower quartile of plasma LA levels had higher serum LDL cholesterol levels. Finally, in our cohort, plasma LA and serum LDL cholesterol levels seemed to have an inverted U-shaped relationship (Figure 2), which does not appear biological plausible. Thus, we speculate that this association might have been confounded by factors we have not been able to identify and adjust for in our analyses.

Plasma LA levels were not associated with serum HDL cholesterol levels, consistent with a recent meta-analysis of clinical trials, reporting no effect of LA intake on HDL cholesterol (39). 


\section{Plasma LA levels and cIMT, blood pressure and kidney function}

cIMT is a validated surrogate marker of future CV events (40). The association between serum LA and cIMT was evaluated in a Spanish population, with a high background consumption of vegetable oils as a part of the Mediterranean diet (41). In that cross-sectional study, serum LA levels were not associated with cIMT. Similarly, in our cohort, with a low background intake of LA, we did not find an association with cIMT. Furthermore, we found only a weak association between plasma LA levels and blood pressure, and no association with serum HDL cholesterol levels, which are considered strong predictors of cIMT progression $(42,43)$. Finally, a recent meta-analysis reported no effect on blood pressure from LA intake (39).

Data on LA intake and kidney function are scarce. A prospective cohort study found less decline in renal function at 3-year follow-up in participants with higher plasma LA levels at baseline (44). In the present study, plasma LA levels were not associated with eGFR <60 $\mathrm{ml} / \mathrm{min} / 1.73 \mathrm{~m}^{2}$, suggesting no benefits for renal function in participants with chronic kidney disease stages 3-5.

\section{Plasma LA levels and general health and lifestyle}

Participants in the upper quartile of plasma LA levels were generally healthier than the remaining cohort, with a lower prevalence of hypertension, hypercholesterolemia, DM, obesity, cerebrovascular and coronary artery disease. This was also reflected by a lower use of antihypertensive-, lipid-lowering and glucose-lowering medications among these participants. Plasma LA levels were not associated with factors such as smoking habits or physical activity, and associations with $\mathrm{CV}$ risk factors remained unchanged when participants were stratified according to educational level and alcohol consumption. Thus, the findings signal that high plasma LA levels may favorably influence CV risk factors. High plasma LA levels 
were associated with overall healthier lifestyle producing a confounder effect, for which we attempted to adjust for in the statistical analysis. However, residual confounding from variables not included in the regression models might still have influenced results, leading to a possible overestimation of associations between plasma LA levels and CV risk factors. Low plasma LA levels have previously been associated with higher BMI, fasting plasma glucose, triglycerides and alcohol consumption (45). Similar findings were reported in statin users compared to non-users (46). A possible explanation could be altered plasma fatty acid composition in individuals with adverse metabolic risk profile, in which case plasma LA level might serve as a marker of cardiometabolic health. Thus, in the present study, we cannot rule out the possibility that our findings have been influenced by revers causality bias.

\section{$\underline{\text { Strengths and limitations }}$}

In this study we measured plasma LA levels as an estimate of LA intake, which is a more reliable marker than self-reported intake from dietary interviews, which are often influenced by recall bias. To our knowledge, this is the largest cohorts in a Norwegian general population where plasma LA levels have been measured by gas chromatography. The study population was well-described and had little missing data. In addition, all participants were born in 1950, thus removing age as a confounding factor.

There are also several limitations. The cross-sectional study design does not allow for any conclusion on causality. Dietary data on LA consumption was not available for this study. Plasma phospholipid PUFA levels do not represent long-term fatty acid intake as accurately as erythrocyte membrane or adipose tissue PUFA levels. However, for LA level estimates, plasma is an equally representative compartment as erythrocyte membrane or adipose tissue (14). Despite careful adjustments for covariates, we cannot rule out residual confounding influencing associations between plasma LA levels and CV risk factors. Furthermore, we 
acknowledge that our results are characterized by weak regression coefficients and that statistically significant associations between plasma LA levels and CV risk factors might have been driven by the large sample size. However, for the purpose of proposing hypothesis regarding the impact of LA consumption on $\mathrm{CV}$ health, we consider our results to be applicable to a Nordic general population. Our results might not apply to other regions, especially those with a high LA consumption. Finally, as all the participants in the ACE 1950 Study were born in 1950, our results might not apply to other age groups.

\section{Conclusion}

We found favorable associations between high plasma LA levels and various CV risk factors in this cross-sectional study of Norwegian individuals born in 1950 .

Well-designed clinical trials are needed to study the effect of an increased LA intake on CV risk factors. 


\section{Acknowledgements}

We acknowledge the skilled study staff at the Clinical Trial Unit, Division of Medicine, Akershus University Hospital, and the Department of Medical Research, Bærum Hospital, Vestre Viken Hospital Trust.

We also thank the staff at The Lipid Research Laboratory, Aalborg University Hospital, Denmark, who performed the fatty acid analyses.

Finally, we would like to thank the study participants.

\section{Disclosures}

The authors declare no disclosures or conflict of interest.

The results presented in this paper have not been published previously and is not under consideration for publication anywhere else.

\section{Author Contributions}

A.C, I.A.E and M.S designed the present study.

H.R, T.O, M.N.L, T.B and A.T designed and organized the ACE 1950 Study including baseline examinations and data collection.

T.V, H.I.-H, E.B.O and O.M.R performed carotid ultrasound and baseline examinations.

E.B.S was responsible for the fatty acid analyses.

A.C, I.A.E and S.N analysed the data.

A.C, I.A.E and M.S edited the manuscript, E.B.S, T.O, H.R, T.V, H.I.-H, S.N, E.B.O, O.M.R, M.N.L, T.B and A.T co-edited the manuscript.

All the authors approved the final version of the manuscript.

A.C submitted the manuscript. 


\section{Funding}

The ACE (Akershus Cardiac Examination) 1950 Study is funded by two health trusts

(Akershus University Hospital HF and Vestre Viken HF), and the South-Eastern Norway

Regional Health Authority, the University of Oslo, and the Norwegian Health Association.

A.C was supported by public funding grants from Akershus University Hospital. 


\section{References:}

1. Whelan J, Fritsche K. Linoleic acid. Adv Nutr. 2013;4(3):311-2.

2. Farvid MS, Ding M, Pan A, Sun Q, Chiuve SE, Steffen LM, et al. Dietary linoleic acid and risk of coronary heart disease: a systematic review and meta-analysis of prospective cohort studies. Circulation. 2014;130(18):1568-78.

3. Wu JH, Lemaitre RN, King IB, Song X, Psaty BM, Siscovick DS, et al. Circulating omega- 6 polyunsaturated fatty acids and total and cause-specific mortality: the Cardiovascular Health Study. Circulation. 2014;130(15):1245-53.

4. Marklund M, Wu JHY, Imamura F, Del Gobbo LC, Fretts A, de Goede J, et al. Biomarkers of Dietary Omega-6 Fatty Acids and Incident Cardiovascular Disease and Mortality. Circulation. 2019;139(21):2422-36.

5. Ramsden CE, Zamora D, Leelarthaepin B, Majchrzak-Hong SF, Faurot KR, Suchindran CM, et al. Use of dietary linoleic acid for secondary prevention of coronary heart disease and death: evaluation of recovered data from the Sydney Diet Heart Study and updated meta-analysis. BMJ. 2013;346:e8707.

6. Ramsden CE, Zamora D, Majchrzak-Hong S, Faurot KR, Broste SK, Frantz RP, et al. Re-evaluation of the traditional diet-heart hypothesis: analysis of recovered data from Minnesota Coronary Experiment (1968-73). BMJ. 2016;353:i1246.

7. Fritsche KL. The science of fatty acids and inflammation. Adv Nutr. 2015;6(3):293S$301 S$.

8. Harris WS, Mozaffarian D, Rimm E, Kris-Etherton P, Rudel LL, Appel LJ, et al. Omega-6 fatty acids and risk for cardiovascular disease: a science advisory from the American Heart Association Nutrition Subcommittee of the Council on Nutrition, Physical Activity, and Metabolism; Council on Cardiovascular Nursing; and Council on Epidemiology and Prevention. Circulation. 2009;119(6):902-7. 
9. Legrand P, Morise A, Kalonji E. Update of French nutritional recommendations for fatty acids. World Rev Nutr Diet. 2011;102:137-43.

10. EFSA. Labelling reference intake values for n-3 and n-6 polyunsaturated fatty acids. EFSA Journal. 2009;1176:1-11.

11. Sioen I, van Lieshout L, Eilander A, Fleith M, Lohner S, Szommer A, et al. Systematic Review on N-3 and N-6 Polyunsaturated Fatty Acid Intake in European Countries in Light of the Current Recommendations - Focus on Specific Population Groups. Ann Nutr Metab. 2017;70(1):39-50.

12. Engeset D, Alsaker E, Ciampi A, Lund E. Dietary patterns and lifestyle factors in the Norwegian EPIC cohort: the Norwegian Women and Cancer (NOWAC) study. Eur J Clin Nutr. 2005;59(5):675-84.

13. Ministers NCo. Nordic Nutrition Recommendations 20122014.

14. Hodson L, Skeaff CM, Fielding BA. Fatty acid composition of adipose tissue and blood in humans and its use as a biomarker of dietary intake. Prog Lipid Res. 2008;47(5):34880.

15. Hodge AM, Simpson JA, Gibson RA, Sinclair AJ, Makrides M, O'Dea K, et al. Plasma phospholipid fatty acid composition as a biomarker of habitual dietary fat intake in an ethnically diverse cohort. Nutr Metab Cardiovasc Dis. 2007;17(6):415-26.

16. Berge T, Vigen T, Pervez MO, Ihle-Hansen H, Lyngbakken MN, Omland T, et al. Heart and Brain Interactions--the Akershus Cardiac Examination (ACE) 1950 Study Design. Scand Cardiovasc J. 2015;49(6):308-15.

17. Obesity: preventing and managing the global epidemic. Report of a WHO consultation. World Health Organ Tech Rep Ser. 2000;894:i-xii, 1-253.

18. Expert Panel on Detection E, Treatment of High Blood Cholesterol in A. Executive Summary of The Third Report of The National Cholesterol Education Program (NCEP) 
Expert Panel on Detection, Evaluation, And Treatment of High Blood Cholesterol In Adults (Adult Treatment Panel III). JAMA. 2001;285(19):2486-97.

19. Levey AS, Stevens LA, Schmid CH, Zhang YL, Castro AF, 3rd, Feldman HI, et al. A new equation to estimate glomerular filtration rate. Ann Intern Med. 2009;150(9):604-12. 20. Ihle-Hansen H, Vigen T, Ihle-Hansen H, Ronning OM, Berge T, Thommessen B, et al. Prevalence of Carotid Plaque in a 63- to 65-Year-Old Norwegian Cohort From the General Population: The ACE (Akershus Cardiac Examination) 1950 Study. J Am Heart Assoc. $2018 ; 7(10)$.

21. Folch J, Lees M, Sloane Stanley GH. A simple method for the isolation and purification of total lipides from animal tissues. J Biol Chem. 1957;226(1):497-509.

22. Burdge GC, Wright P, Jones AE, Wootton SA. A method for separation of phosphatidylcholine, triacylglycerol, non-esterified fatty acids and cholesterol esters from plasma by solid-phase extraction. Br J Nutr. 2000;84(5):781-7.

23. Wu JHY, Marklund M, Imamura F, Tintle N, Ardisson Korat AV, de Goede J, et al. Omega- 6 fatty acid biomarkers and incident type 2 diabetes: pooled analysis of individuallevel data for 39740 adults from 20 prospective cohort studies. Lancet Diabetes Endocrinol. 2017;5(12):965-74.

24. Cabout M, Alssema M, Nijpels G, Stehouwer CDA, Zock PL, Brouwer IA, et al. Circulating linoleic acid and alpha-linolenic acid and glucose metabolism: the Hoorn Study. Eur J Nutr. 2017;56(6):2171-80.

25. Imamura F, Micha R, Wu JH, de Oliveira Otto MC, Otite FO, Abioye AI, et al. Effects of Saturated Fat, Polyunsaturated Fat, Monounsaturated Fat, and Carbohydrate on GlucoseInsulin Homeostasis: A Systematic Review and Meta-analysis of Randomised Controlled Feeding Trials. PLoS Med. 2016;13(7):e1002087. 
26. Knottnerus SJG, Bleeker JC, Wust RCI, Ferdinandusse S, L IJ, Wijburg FA, et al. Disorders of mitochondrial long-chain fatty acid oxidation and the carnitine shuttle. Rev Endocr Metab Disord. 2018;19(1):93-106.

27. Liu X, Li Y, Tobias DK, Wang DD, Manson JE, Willett WC, et al. Changes in Types of Dietary Fats Influence Long-term Weight Change in US Women and Men. J Nutr. 2018;148(11):1821-9.

28. Nakamura MT, Nara TY. Structure, function, and dietary regulation of delta6, delta5, and delta9 desaturases. Annu Rev Nutr. 2004;24:345-76.

29. Warensjö E, Risérus U, Vessby B. Fatty acid composition of serum lipids predicts the development of the metabolic syndrome in men. Diabetologia. 2005;48(10):1999-2005. 30. Vessby B, Gustafsson IB, Tengblad S, Boberg M, Andersson A. Desaturation and elongation of Fatty acids and insulin action. Ann N Y Acad Sci. 2002;967:183-95.

31. Hussein N, Ah-Sing E, Wilkinson P, Leach C, Griffin BA, Millward DJ. Long-chain conversion of [13C]linoleic acid and alpha-linolenic acid in response to marked changes in their dietary intake in men. J Lipid Res. 2005;46(2):269-80.

32. Rett BS, Whelan J. Increasing dietary linoleic acid does not increase tissue arachidonic acid content in adults consuming Western-type diets: a systematic review. Nutr Metab (Lond). $2011 ; 8: 36$

33. Johnson GH, Fritsche K. Effect of dietary linoleic acid on markers of inflammation in healthy persons: a systematic review of randomized controlled trials. J Acad Nutr Diet. 2012;112(7):1029-41, 41 e1-15.

34. Kelley-Hedgepeth A, Lloyd-Jones DM, Colvin A, Matthews KA, Johnston J, Sowers MR, et al. Ethnic differences in C-reactive protein concentrations. Clin Chem. 2008;54(6):1027-37. 
35. Brenna JT. Efficiency of conversion of alpha-linolenic acid to long chain n-3 fatty acids in man. Curr Opin Clin Nutr Metab Care. 2002;5(2):127-32.

36. Mensink RP, Zock PL, Kester AD, Katan MB. Effects of dietary fatty acids and carbohydrates on the ratio of serum total to HDL cholesterol and on serum lipids and apolipoproteins: a meta-analysis of 60 controlled trials. Am J Clin Nutr. 2003;77(5):1146-55. 37. Drouin-Chartier JP, Tremblay AJ, Lepine MC, Lemelin V, Lamarche B, Couture P. Substitution of dietary omega- 6 polyunsaturated fatty acids for saturated fatty acids decreases LDL apolipoprotein B-100 production rate in men with dyslipidemia associated with insulin resistance: a randomized controlled trial. Am J Clin Nutr. 2018;107(1):26-34.

38. van Schalkwijk DB, Pasman WJ, Hendriks HF, Verheij ER, Rubingh CM, van Bochove K, et al. Dietary medium chain fatty acid supplementation leads to reduced VLDL lipolysis and uptake rates in comparison to linoleic acid supplementation. PLoS One. 2014;9(7):e100376.

39. Al-Khudairy L, Hartley L, Clar C, Flowers N, Hooper L, Rees K. Omega 6 fatty acids for the primary prevention of cardiovascular disease. Cochrane Database Syst Rev. 2015(11):CD011094.

40. Polak JF, O'Leary DH. Carotid Intima-Media Thickness as Surrogate for and Predictor of CVD. Glob Heart. 2016;11(3):295-312 e3.

41. Sala-Vila A, Cofan M, Perez-Heras A, Nunez I, Gilabert R, Junyent M, et al. Fatty acids in serum phospholipids and carotid intima-media thickness in Spanish subjects with primary dyslipidemia. Am J Clin Nutr. 2010;92(1):186-93.

42. Ren L, Cai J, Liang J, Li W, Sun Z. Impact of Cardiovascular Risk Factors on Carotid Intima-Media Thickness and Degree of Severity: A Cross-Sectional Study. PLoS One. 2015;10(12):e0144182. 
43. Touboul PJ, Labreuche J, Bruckert E, Schargrodsky H, Prati P, Tosetto A, et al. HDLC, triglycerides and carotid IMT: a meta-analysis of 21,000 patients with automated edge detection IMT measurement. Atherosclerosis. 2014;232(1):65-71.

44. Lauretani F, Semba RD, Bandinelli S, Miller ER, 3rd, Ruggiero C, Cherubini A, et al. Plasma polyunsaturated fatty acids and the decline of renal function. Clin Chem. 2008;54(3):475-81.

45. Pertiwi K, Wanders AJ, Harbers MC, Küpers LK, Soedamah-Muthu SS, de Goede J, et al. Plasma and Dietary Linoleic Acid and 3-Year Risk of Type 2 Diabetes After Myocardial Infarction: A Prospective Analysis in the Alpha Omega Cohort. Diabetes Care. 2020;43(2):358-65.

46. Pertiwi K, Kok DE, Wanders AJ, de Goede J, Zock PL, Geleijnse JM. Circulating n-3 fatty acids and linoleic acid as indicators of dietary fatty acid intake in post-myocardial infarction patients. Nutr Metab Cardiovasc Dis. 2019;29(4):343-50. 


\section{Figure legends}

\section{Figure 1}

Flowchart of the inclusion of study participants.

\section{Figure 2}

Univariate associations between plasma linoleic acid levels (x-axis) and CV risk factors (yaxis), modeled as restricted cubic spline. We used three knots, placed at $10^{\text {th }}, 50^{\text {th }}$ and $90^{\text {th }}$ percentile of plasma linoleic acid levels, measured in weight percentage of total plasma phospholipid fatty acids. 
Table 1. Characteristics of study participants according to quartiles of plasma linoleic acid levels.

\begin{tabular}{|c|c|c|c|c|c|c|}
\hline & All patients & Quartile 1 & Quartile 2 & Quartile 3 & Quartile 4 & $\mathbf{p}$ \\
\hline Linoleic acid level, wt\% & 11.4-32.0 & $\leq 18.74$ & $18.75-20.82$ & 20.83-22.77 & $\geq 22.78$ & \\
\hline Number of participants & 3683 & 922 & 919 & 923 & 919 & \\
\hline Age, months & $767(7.8)$ & $768(7.9)$ & $767(7.7)$ & $767(7.9)$ & $766(7.4)$ & $<0.001$ \\
\hline Gender (Male), \% & 51.3 & 54.0 & 49.2 & 49.3 & 52.8 & 0.08 \\
\hline Daily fruit intake, $\%$ & 51.2 & 51.2 & 52.3 & 50.6 & 50.7 & 0.89 \\
\hline Daily vegetable intake, $\%$ & 58.9 & 58.6 & 58.5 & 60.8 & 57.9 & 0.61 \\
\hline $\begin{array}{l}\text { Daily whole wheat bread } \\
\text { intake, } \%\end{array}$ & 57.3 & 58.3 & 55.9 & 57.7 & 57.4 & 0.76 \\
\hline EPA, $w t \%$ & $2.6(1.4)$ & $3.4(1.7)$ & $2.8(1.2)$ & $2.3(1.1)$ & $1.9(0.9)$ & $<0.001$ \\
\hline DHA, $w t \%$ & $5.5(1.4)$ & $6.2(1.6)$ & $5.7(1.4)$ & $5.3(1.2)$ & $4.8(1.2)$ & $<0.001$ \\
\hline $\mathrm{ALA}, w t \%$ & $0.26(0.1)$ & $0.22(0.1)$ & $0.25(0.1)$ & $0.27(0.1)$ & $0.29(0.1)$ & $<0.001$ \\
\hline $\mathrm{AA}, w t \%$ & $9.2(2.1)$ & $10.3(2.4)$ & $9.6(1.9)$ & $8.9(1.6)$ & $8.1(1.6)$ & $<0.001$ \\
\hline Current smoker, $\%$ & 14.5 & 16.7 & 13.2 & 15.0 & 13.0 & 0.10 \\
\hline $\begin{array}{l}\text { Alcohol consumption, }(\geq 2 \\
\text { times weekly), } \%\end{array}$ & 47.4 & 51.8 & 49.1 & 46.2 & 42.3 & $<0.001$ \\
\hline $\begin{array}{l}\text { Physical activity ( } \geq 2 \text { times } \\
\text { weekly), } \%\end{array}$ & 61.7 & 58.9 & 62.6 & 63.2 & 62.3 & 0.22 \\
\hline Higher education, $\%$ & 46.5 & 42.8 & 43.5 & 48.1 & 51.6 & $<0.001$ \\
\hline $\begin{array}{l}\text { Systolic blood pressure, } \\
\mathrm{mmHg}\end{array}$ & $138(19)$ & 139 (19) & 139 (19) & $137(18)$ & $137(19)$ & 0.01 \\
\hline $\begin{array}{l}\text { Diastolic blood pressure, } \\
\mathrm{mmHg}\end{array}$ & $77(10)$ & $78(10)$ & $77(10)$ & $77(10)$ & $76(10)$ & 0.11 \\
\hline Total cholesterol, $\mathrm{mmol} / \mathrm{L}$ & $5.4(1.1)$ & $5.2(1.2)$ & $5.5(1.1)$ & $5.6(1.1)$ & $5.5(1.0)$ & $<0.001$ \\
\hline HDL cholesterol, $\mathrm{mmol} / \mathrm{L}$ & $1.5(0.5)$ & $1.5(0.5)$ & $1.5(0.5)$ & $1.6(0.5)$ & $1.6(0.5)$ & $<0.001$ \\
\hline LDL cholesterol, $\mathrm{mmol} / \mathrm{L}$ & $3.3(1.0)$ & $3.0(1.0)$ & $3.4(1.0)$ & $3.4(0.9)$ & $3.4(0.9)$ & $<0.001$ \\
\hline Triglycerides, $\mathrm{mmol} / \mathrm{L}$ & $\begin{array}{c}1.2 \\
(0.4-2.0)\end{array}$ & $\begin{array}{c}1.3 \\
(0.3-2.8)\end{array}$ & $\begin{array}{c}1.2 \\
(0.4-2.0)\end{array}$ & $\begin{array}{c}1.2 \\
(0.5-1.9)\end{array}$ & $\begin{array}{c}1.0 \\
(0.3-1.7)\end{array}$ & $<0.001$ \\
\hline Fasting plasma glucose, $\mathrm{mmol} / \mathrm{L}$ & $\begin{array}{c}5.3 \\
(4.5-6.2)\end{array}$ & $\begin{array}{c}5.5 \\
(4.5-6.5)\end{array}$ & $\begin{array}{c}5.3 \\
(4.4-6.2)\end{array}$ & $\begin{array}{c}5.3 \\
(4.5-6.1)\end{array}$ & $\begin{array}{c}5.2 \\
(4.4-6.0)\end{array}$ & $<0.001$ \\
\hline $\mathrm{HbA} 1 \mathrm{c}, \%$ & $\begin{array}{c}5.7 \\
(5.3-6.1)\end{array}$ & $\begin{array}{c}5.7 \\
(5.2-6.2)\end{array}$ & $\begin{array}{c}5.7 \\
(5.3-6.1)\end{array}$ & $\begin{array}{c}5.7 \\
(5.3-6.1)\end{array}$ & $\begin{array}{c}5.7 \\
(5.3-6.1)\end{array}$ & $<0.001$ \\
\hline
\end{tabular}




\begin{tabular}{|c|c|c|c|c|c|c|}
\hline $\begin{array}{l}\text { Body mass index (BMI), } \\
\mathrm{kg} / \mathrm{m}^{2}\end{array}$ & $27.1(4.4)$ & $28.1(4.4)$ & $27.3(4.3)$ & $27.1(4.5)$ & $26.0(4.3)$ & $<0.001$ \\
\hline eGFR, $\mathrm{ml} / \mathrm{min} \times 1.73 \mathrm{~m} 2$ & $83(12.0)$ & $84(12.3)$ & $83(11.8)$ & $84(12.0)$ & $82(11.5)$ & 0.008 \\
\hline cIMT, $m m$ & $0.73(0.1)$ & $0.74(0.1)$ & $0.73(0.1)$ & $0.73(0.1)$ & $0.72(0.1)$ & 0.01 \\
\hline $\mathrm{CRP}, m g / L$ & $\begin{array}{c}1.5 \\
(1.5-1.5)\end{array}$ & $\begin{array}{c}1.5 \\
(1.5-1.5)\end{array}$ & $\begin{array}{c}1.5 \\
(1.5-1.5)\end{array}$ & $\begin{array}{c}1.5 \\
(1.5-1.5)\end{array}$ & $\begin{array}{c}1.5 \\
(1.5-1.5)\end{array}$ & 0.07 \\
\hline Hypertension, $\%$ & 62.0 & 72.6 & 62.5 & 59.5 & 53.3 & $<0.001$ \\
\hline Hypercholesterolemia, \% & 52.6 & 71.0 & 58.2 & 47.1 & 33.9 & $<0.001$ \\
\hline Cerebrovascular disease, $\%$ & 3.7 & 5.1 & 4.1 & 4.1 & 1.6 & $<0.001$ \\
\hline Coronary artery disease, $\%$ & 7.0 & 13.7 & 6.7 & 4.9 & 2.8 & $<0.001$ \\
\hline Diabetes mellitus, $\%$ & 8.5 & 12.9 & 9.4 & 6.3 & 5.4 & $<0.001$ \\
\hline Obesity (BMI $\geq 30), \%$ & 22.6 & 28.5 & 24.4 & 22.2 & 15.1 & $<0.001$ \\
\hline $\begin{array}{l}\text { CKD stages } 3-5(\text { eGFR }<60 \\
\left.\mathrm{ml} / \mathrm{min} \times 1.73 \mathrm{~m}^{2}\right), \%\end{array}$ & 3.9 & 3.9 & 3.6 & 3.4 & 4.6 & 0.58 \\
\hline \multicolumn{7}{|l|}{ Medication, $\%$} \\
\hline Diuretics & 3.1 & 4.3 & 3.2 & 2.4 & 2.4 & 0.05 \\
\hline Beta blockers & 13.4 & 20.8 & 13.2 & 11.4 & 8.1 & $<0.001$ \\
\hline Calcium channel blockers & 8.1 & 12.1 & 7.7 & 8.8 & 3.9 & $<0.001$ \\
\hline ACEi or ARB & 26.9 & 36.2 & 26.3 & 25.6 & 19.6 & $<0.001$ \\
\hline Lipid-lowering drugs & 26.1 & 49.9 & 27.9 & 17.2 & 9.6 & $<0.001$ \\
\hline Glucose-lowering drugs & 5.2 & 8.2 & 4.8 & 4.5 & 3.2 & $<0.001$ \\
\hline
\end{tabular}

Results are presented as percentage for categorical data and mean value (standard deviation) for continuous data. Differences between groups were evaluated using Chi square for dichotomous data, the Kruskal-Wallis test for triglycerides, fasting plasma glucose, HbA1c and CRP, and ANOVA for other continuous data.

Abbreviations: EPA: Eicosapentaenoic acid. DHA: Docosahexaenoic acid. ALA: Alphalinoleic acid. AA: Arachidonic acid. HDL: High density lipoprotein. LDL: Low density lipoproteins HbA1c: Hemoglobin A1c. BMI: Body mass index. eGFR: Estimated glomerular filtration rate (CKD-EPI formula). cIMT: Carotid intima-media thickness. CRP: C-reactive protein. CKD: Chronic kidney disease. ACEi: Angiotensin converting enzyme inhibitor. ARB: Angiotensin receptor blocker. 
Table 2. Associations between plasma linoleic acid levels and cardiovascular risk factors.

\begin{tabular}{|c|c|c|c|c|c|}
\hline \multicolumn{6}{|c|}{ Univariable linear regression analysis } \\
\hline Cardiovascular risk factors & $\mathbf{n}$ & Unstd. $\beta$-coeff. $(95 \%$ CI) & Std. $\beta$-coeff. & $\mathbf{p}$ & $\mathbf{R}^{2}$ \\
\hline HDL cholesterol, $\mathrm{mmol} / \mathrm{L}$ & 3680 & $0.01(0.01,0.02)$ & 0.08 & $<0.001$ & 0.01 \\
\hline LDL cholesterol, $\mathrm{mmol} / \mathrm{L}$ & 3657 & $0.05(0.04,0.06)$ & 0.14 & $<0.001$ & 0.02 \\
\hline Triglycerides, $\mathrm{mmol} / \mathrm{L}$ & 3680 & $-0.04(-0.05,-0.03)$ & -0.18 & $<0.001$ & 0.03 \\
\hline Fasting glucose, $\mathrm{mmol} / \mathrm{L}$ & 3675 & $-0.06(-0.07,-0.05)$ & -0.18 & $<0.001$ & 0.03 \\
\hline $\mathrm{HbA} 1 \mathrm{c}, \%$ & 3669 & $-0.02(-0.02,-0.01)$ & -0.08 & $<0.001$ & 0.01 \\
\hline $\mathrm{BMI}, \mathrm{kg} / \mathrm{m}^{2}$ & 3683 & $-0.27(-0.31,-0.23)$ & -0.18 & $<0.001$ & 0.03 \\
\hline $\mathrm{SBP}, m m H g$ & 3679 & $-0.40(-0.61,-0.20)$ & -0.06 & $<0.001$ & 0.004 \\
\hline $\mathrm{DBP}, m m H g$ & 3679 & $-0.12(-0.23,-0.01)$ & -0.04 & 0.03 & 0.001 \\
\hline $\mathrm{eGFR}, \mathrm{ml} / \mathrm{min} \times 1.73 \mathrm{~m} 2$ & 3664 & $-0.21(-0.34,-0.07)$ & -0.05 & 0.002 & 0.002 \\
\hline cIMT, $m m$ & 3661 & $-0.002(-0.003,-0.001)$ & -0.05 & 0.002 & 0.002 \\
\hline $\mathrm{CRP}, m g / L$ & 3669 & $-1.01(-1.02,-1.01)$ & -0.06 & $<0.001$ & 0.003 \\
\hline \multicolumn{6}{|c|}{ Multivariable linear regression analysis } \\
\hline Cardiovascular risk factors & $\mathbf{n}$ & Unstd. $\beta$-coeff. $(95 \%$ CI $)$ & Std. $\beta$-coeff. & $\mathbf{p}$ & $\mathbf{R}^{2}$ \\
\hline HDL cholesterol, $\mathrm{mmol}^{\mathrm{a}} \mathrm{L}^{\mathrm{a}}$ & 3609 & $0.0001(-0.005,0.005)$ & 0.00 & 0.98 & 0.30 \\
\hline LDL cholesterol, $\mathrm{mmol}^{\mathrm{L}} \mathrm{L}^{\mathrm{b}}$ & 3646 & $-0.01(-0.02,-0.002)$ & -0.04 & 0.02 & 0.28 \\
\hline Triglycerides, $\mathrm{mmol} / \mathrm{L}^{\mathrm{c}}$ & 3609 & $-0.02(-0.03,-0.02)$ & -0.10 & $<0.001$ & 0.19 \\
\hline Fasting glucose, $\mathrm{mmol} / \mathrm{L}^{\mathrm{d}}$ & 3605 & $-0.03(-0.04,-0.02)$ & -0.10 & $<0.001$ & 0.36 \\
\hline $\mathrm{HbA} 1 \mathrm{c}, \%^{\mathrm{e}}$ & 3599 & $0.00(-0.01,0.01)$ & -0.002 & 0.90 & 0.41 \\
\hline BMI, $k g / m^{\mathrm{f}}$ & 3612 & $-0.20(-0.25,-0.15)$ & -0.13 & $<0.001$ & 0.16 \\
\hline $\mathrm{SBP}^{\mathrm{g}}$ & 3609 & $-0.25(-0.47,-0.03)$ & -0.04 & 0.03 & 0.03 \\
\hline $\mathrm{DBP}^{\mathrm{h}}$ & 3609 & $-0.14(-0.25,-0.02)$ & -0.02 & 0.02 & 0.12 \\
\hline $\mathrm{eGFR}, \mathrm{ml} / \min \times 1.73 m 2^{\mathrm{i}}$ & 3593 & $-0.37(-0.51,-0.23)$ & -0.09 & $<0.001$ & 0.04 \\
\hline $\operatorname{cIMT}, m m^{\mathrm{j}}$ & 3635 & $0.000(-0.001,0.001)$ & -0.01 & 0.73 & 0.07 \\
\hline $\mathrm{CRP}, m g / L^{\mathrm{k}}$ & 3603 & $-1.01(-1.02,1.00)$ & -0.03 & 0.06 & 0.07 \\
\hline
\end{tabular}

${ }^{a}$ Gender, smoking, diabetes mellitus, BMI, lipid-lowering drugs, glucose-lowering drugs, hypertension, physical activity, higher education, age (months), alcohol consumption.

${ }^{\mathrm{b}}$ Gender, diabetes mellitus, BMI, lipid-lowering drugs, glucose-lowering drugs, higher education, age (months).

${ }^{\mathrm{c}}$ Gender, smoking, diabetes mellitus, BMI, lipid-lowering drugs, glucose-lowering drugs, hypertension, physical activity, higher education, age (months), alcohol consumption.

${ }^{\mathrm{d}}$ Gender, smoking, BMI, lipid-lowering drugs, glucose-lowering drugs, hypertension, physical activity, higher education, age (months), alcohol consumption.

${ }^{\mathrm{e}}$ Gender, smoking, BMI, lipid-lowering drugs, glucose-lowering drugs, hypertension, physical activity, higher education, age (months), alcohol consumption.

${ }^{\mathrm{f}}$ Gender, smoking, diabetes mellitus, lipid-lowering drugs, hypertension, physical activity, higher education, age (months), alcohol consumption.

g Gender, smoking, diabetes mellitus, BMI, lipid-lowering drugs, physical activity, higher education, age (months), alcohol consumption, antihypertensive drugs.

${ }^{\mathrm{h}}$ Gender, smoking, diabetes mellitus, BMI, lipid-lowering drugs, glucose-lowering drugs, physical activity, higher education, age (months), alcohol consumption, antihypertensive drugs.

${ }^{\mathrm{i}}$ Gender, smoking, diabetes mellitus, BMI, lipid-lowering drugs, hypertension, physical activity, higher education, age (months), alcohol consumption. 
${ }^{\mathrm{j}}$ Gender, smoking, BMI, glucose-lowering drugs, hypertension, age (months).

${ }^{\mathrm{k}}$ Gender, smoking, diabetes mellitus, BMI, lipid-lowering drugs, hypertension, physical activity, higher education, age (months).

Unstandardized $\beta$ coefficients (Unstd. $\beta$-coeff.) with corresponding 95\% confidence intervals $(\mathrm{CI})$, standardized $\beta$ coefficients (Std. $\beta$-coeff.), $\mathrm{p}$-values and explained variance $\left(\mathrm{R}^{2}\right)$ are given for plasma LA in the univariable analysis and for the fully adjusted multivariable model.

Abbreviations: HDL: High-density lipoprotein. LDL: Low-density lipoprotein. HbA1c: Hemoglobin A1c. BMI: Body mass index. SBP: Systolic blood pressure. DBP: Diastolic blood pressure. eGFR: Estimated glomerular filtration rate. cIMT: Carotid intima-media thickness. CRP: C-reactive protein. 
Fig. 1

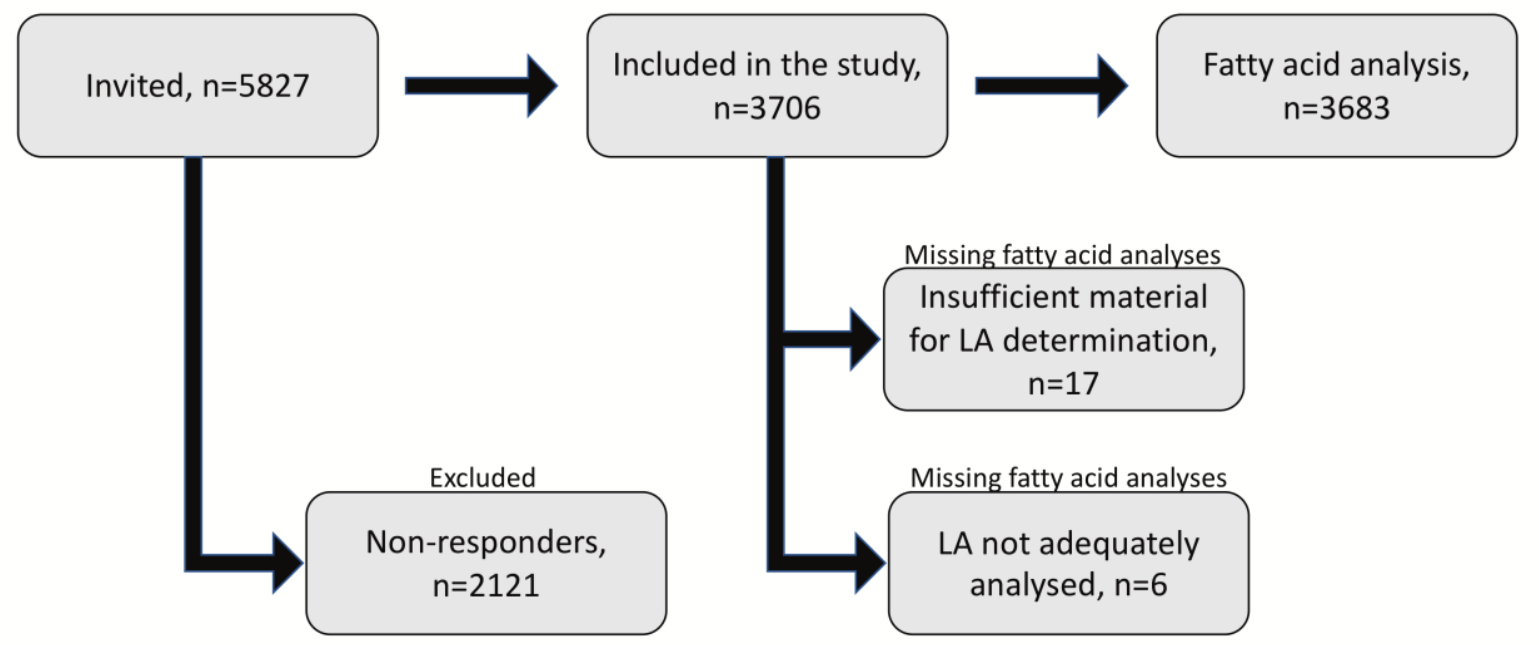


Fig 2.
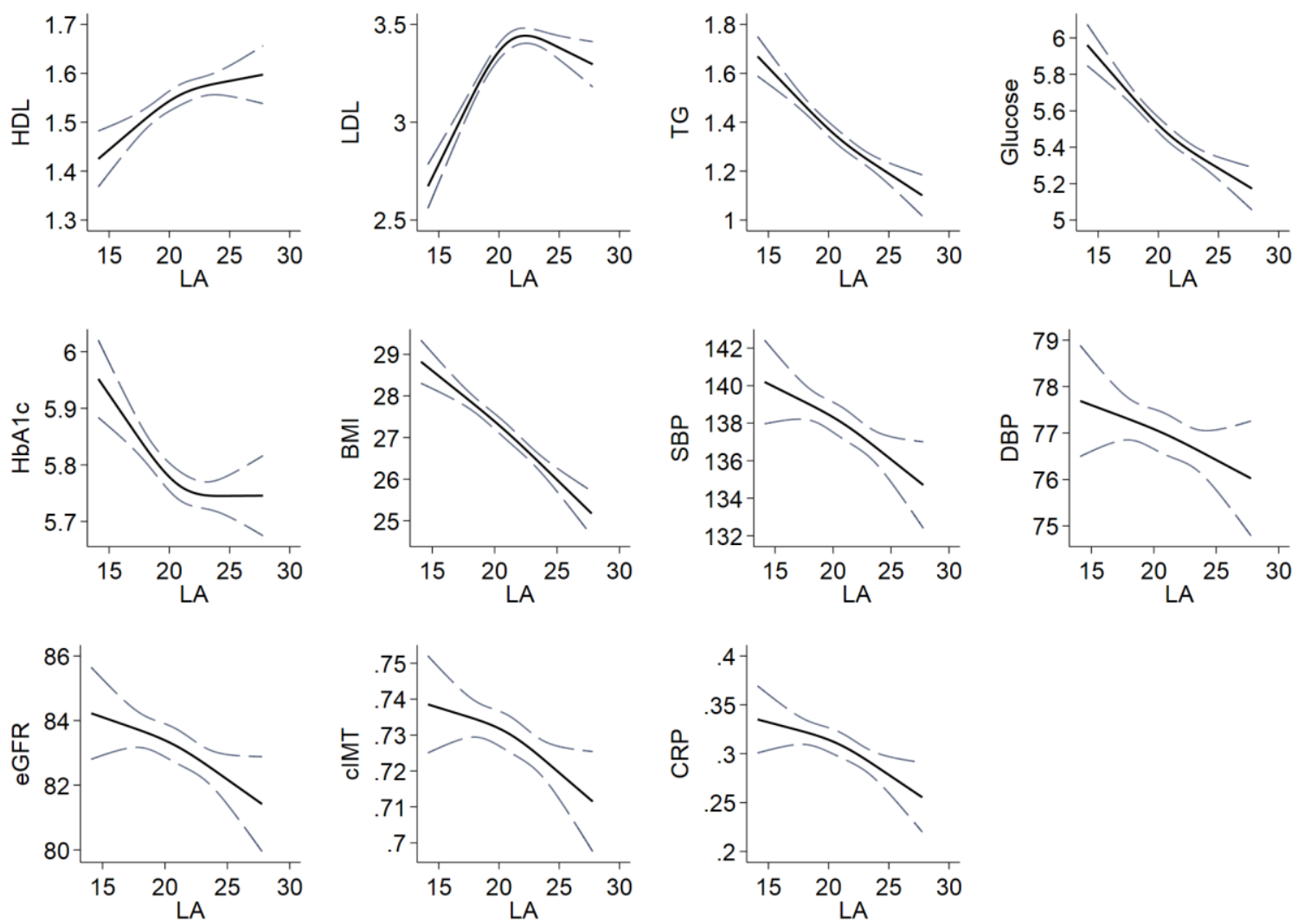